\title{
Note to Authors
}

At present, international requirements call for strict adherence to ethical principles in the course of research and in reporting the respective results in scientific publications. In this regard, our journal, starting from this issue (No. 3 of Vol. 45, 2013), includes in each paper a special separate paragraph, "Compliance with Ethical Standards," which is placed after the main text before the list of references. The following points should be clearly indicated in this paragraph:

- Experiments on animals and/or tests on humans were carried out in accordance with the provisions of the Helsinki Declaration (1975, revised and supplemented in 2005) and with the directives of the National Committees for Research Ethics; the experiments were approved by the Ethics Committee of the institution in which the study was performed;

- Current rules for keeping and using laboratory animals consistent with the principles of the European Convention for the Protection of Vertebrate Animals Used for Experimental and Other Scientific Purposes (Strasbourg, 1985) were observed;

- In tests on humans, all participants provided their written informed consent; if necessary, all measures to ensure the anonymity of the participants were taken;

- There is no conflict of interest between the authors and any individual or organization that may influence their work (their names and initials are listed). A collective statement, e.g., "The authors proclaim that they have no conflict of interest", is not sufficient.

In the same section, additional sources of funding (if any) of the research work for one author or another should be mentioned.

The respective paragraph has been introduced in the "Instructions for Authors" of our journal. 